\title{
Las Leyes de Newton de la mecánica: Una revisión histórica y sus implicaciones en los textos de enseñanza
}

\author{
José Sebastiá M. Sebastiá \\ Departamento de Física \\ Universidad Simón Bolívar (Venezuela)
}

Resumen: Este trabajo destaca la importancia de una presentación coherente y actualizada de la dinámica clasica como un factor clave para su aprendizaje. La primera parte de este trabajo contiene un análisis histórico de los aspectos críticos de las leyes de la dinámica clásica que muestra sus ambigüedades y sus posibles interpretaciones. A continuación, se analizan las alternativas de enseñanza en algunos libros de texto haciendo especial énfasis en sus características fundamentales. Como resultado, se concluye que la presentación de la dinámica clásica, tal como se hace actualmente, muestra deficiencias tanto de coherencia como de actualización, lo que sugiere la necesidad de una revisión general de la misma.

Palabras clave: dinámica clásica; leyes de Newton, transposición didáctica, contenido curricular.

Abstract: This work highlights the importance of a coherent and updated presentation of classical dynamic as a key factor for learning. The first part of this work contains an historical analysis of the critical aspects of the laws of dynamics showing their ambiguities and their possible interpretations. Further on, the teaching alternatives in some textbooks are analyzed making an emphasis in their fundamental characteristics. As a result, we conclude that the presentation of the classic dynamics, as is currently done, shows weaknesses both in coherence and updated, suggesting the need for a general review of it.

Key Words: Classic dynamics; Newton's laws, didactic transposition, content curricular.

(Fecha de recepción: febrero, 2013, y de aceptación: septiembre, 2013)

DOI: 7203/DCES.27.2241 


\section{Introducción}

Pocas áreas de la física han suscitado mayor cantidad de investigaciones en el ámbito de la enseñanza que la relativa a las relaciones entre la fuerza y el movimiento. La mayoría de las investigaciones han puesto de relieve las dificultades que los estudiantes encuentran para la comprensión de conceptos tales como fuerza, masa, inercia, interacción, etc. (Driver, Guesne y Tiberghien, 1985; Thijs y Van Der Berg, 1995; Carrascosa y Gil-Pérez, 1992; Furió, Solbes y Carrascosa, 2006; Solbes y Palomar, 2011). Muchas de estas dificultades de comprensión de los estudiantes han generado nuevas metodologías que propiciasen la sustitución de las "concepciones alternativas" de los estudiantes por aquellas que son pretendidas en la enseñanza (Posner,Strike, Hewson y Gertzog, 1982, Champagne, Gunstone y Klopfer, 1985, Duit, 2003, Gil-Pérez, 1996). Aunque los cambios en los métodos de enseñanza han involucrado algunos cambios en los contenidos expuestos, estos contenidos han seguido en gran parte los patrones existentes. Hestenes (1999) ha enfatizado que parte de estas dificultades de compresión de la mecánica clásica se deben a que los libros de texto no explicitan algunas de las hipótesis básicas de la teoría newtoniana, tales como las concepciones absolutas de espacio y de tiempo, no incorporan en la presentación la noción de campo gravitatorio, no hacen una diferenciación clara entre las nociones intuitivas y las formulaciones científicas, no preparan el camino para la comprensión de la mecánica cuánti- ca y relativista y, en general, no toman en cuenta las profundas modificaciones que la física ha experimentado en los últimos siglos.

Lo que habitualmente se presenta como mecánica clásica es más bien una adaptación de los enunciados originales de Newton con algunas aportaciones de Lagrange, Euler, Mach y Kichhoff. Sin embargo, han pasado más de trescientos años desde su propuesta y muchos de sus supuestos han sido seriamente revisados o totalmente abandonados. A pesar de la célebre frase de Newton "hypotheses non fingo" (no hago hipótesis), lo cierto es que toda su obra contiene múltiples hipótesis de naturaleza epistemológica: concepción absoluta del espacio y del tiempo, fuerzas de acción a distancia, fuerzas de inercia, transmisión instantánea de las fuerzas, masa como cantidad de materia, etc., que son insostenibles en una teoría física actual y posiblemente inapropiadas para su enseñanza.

Chevallard (1991) acuñó el término de "transposición didáctica" para destacar el hecho de que un contenido científico no puede ser simplemente transferido a los estudiantes sin pasar por un proceso de transformación y adaptación a las condiciones particulares de enseñanza. La necesidad de responder adecuadamente a los requerimientos pedagógicos, sociales y políticos obliga a una verdadera reconstrucción del contenido que transforme el "saber científico" (savoir savant) en "saber para enseñar" (savoir enseigné), pero para que tenga lugar esta transformación resulta pertinente proceder a un análisis disciplinar que permita emerger los puntos 
nodales y las estructuras conceptuales y metodológicas de cada argumento. Se requiere, en suma, elaborar una nueva estructura de conocimiento consecuente con la evolución de la ciencia que presente a los estudiantes una visión coherente y actualizada del contenido a enseñar.

En el caso particular de la física, cada contenido tiene unas características epistemológicas propias que dependen de su evolución histórica y del papel que ocupa en el ambito de la física. Partiendo del supuesto de que la selección y elaboración del contenido es un aspecto fundamental que condiciona el aprendizaje, resulta de gran valor retomar una revisión crítica de la estructura conceptual y epistemológica de mecánica clásica y de la transposición didáctica que explicita o implícitamente se está llevando a cabo, para resaltar sus debilidades y fortalezas y permitir, en caso necesario, una revisión de la misma.

Con el objeto de indagar hasta qué punto la presentación de la dinámica clásica que se hace en los textos de enseñanza responde a las características de coherencia y actualización, hemos considerado apropiado hacer una revisión histórica que permita emerger los puntos críticos de la teoría y las modificaciones de formulación que ha experimentado, partiendo de la propuesta inicial newtoniana y pasando por las diferentes aproximaciones que han enriquecido y alterado su formulación original. La revisión de una significativa muestra de textos "clásicos" de física universitaria nos ha permitido, a partir del marco teórico proporcionado por la revisión histórica, caracterizar desde el punto de vista epistemológico algunos esquemas de presentación (pictures) utilizados en los textos, así como constatar que algunos de estos esquemas resultan más propicios para las incorporaciones posteriores de las mecánicas relativista y cuántica.

\section{La formulación de las Leyes de Newton: algunos aspectos críticos}

Nos proponemos en este apartado hacer un breve recuento de algunos aspectos problemáticos de las leyes de Newton, de las observaciones que se han hecho a lo largo de la historia y de las propuestas alternativas que se han elaborado para solventarlas. Consideramos que una adaptación de la mecánica clásica a la enseñanza no puede ignorar algunos elementos críticos que han sido ampliamente debatidos.

\section{La primera ley de Newton}

Newton publicó en 1687 la primera versión del libro Mathematical Principles of Natural Philosophy que engloba toda su teoría de la mecánica expuesta articuladamente mediante ocho definiciones y tres axiomas. Los tres axiomas constituyen lo que hoy conocemos con el nombre de leyes de Newton: la ley de la inercia; la ley de proporcionalidad entre la fuerza resultante y la aceleración; y la tercera ley o principio de acción y reacción.

La primera ley establece que "todo cuerpo persevera en su estado de reposo o movimiento uniforme a lo largo de una línea recta, a menos que una fuerza impresa lo obligue a cambiar su estado" ("Corpus omne perseverare in statu suo 
quiescendi vel movendi uniformiter in directum, nisi quatenus illud a viribus impressis cogitur statum suum mutare”, Principia, p.13).

Dos aspectos destacables en este enunciado son: a) la consideración del "estado" de reposo y de movimiento uniforme en línea recta como equivalentes desde el punto de vista dinámico; b) la suficiencia de la actuación de una fuerza impresa (fuerza externa) para el cambio de estado de reposo o movimiento rectilíneo uniforme. Es importante destacar que Newton distinguía dos tipos de fuerza: la fuerza interna o de inercia, "vis insita" (definición III) y la fuerza impresa o externa, "vis impres$s a$ " (definición IV). La fuerza de inercia justificaba que un cuerpo se resistiese a cambiar su estado de reposo o movimiento rectilíneo uniforme y sólo modificase su estado por la aplicación de una fuerza externa (la fuerza impresa).

Una puntualización posterior debida a Euler (Mechanics or the Science of Motion Presented Analytically, 1736) aclara que: el estado en reposo o de movimiento rectilíneo uniforme de un cuerpo es "el estado de referencia" de la dinámica newtoniana y, por lo tanto, no requiere explicación alguna. Asi pues, la mecánica de Newton abandona la máxima medieval de que "todo lo que se mueve es movido por algo" (omnia quod movetur ab alio movetur) para considerar equivalentes (e incausados) el estado de reposo y el estado de movimiento rectilineo uniforme. Por el contrario, el cambio de movimiento sí que requeriría explicación y la "fuerza impresa" sería la condición necesaria y suficiente para explicarlo.
Según Laplace (Traité de Mécanique Céleste, 1799), la justificación lógica ( $a$ priori) de la ley de la inercia pudiera ser resumida así: "un cuerpo en movimiento seguirá moviéndose en línea recta y a velocidad constante ya que no posee en sí mismo ninguna capacidad interna para desviarse hacia la derecha o hacia la izquierda, ni para aumentar o disminuir su velocidad, por lo tanto se mantendrá con movimiento rectilíneo y uniforme". Jacobi señaló la convencionalidad del argumento anterior(Lectures on Analytical Mechanics, 1847), ya que no es sino una "declaración de principios" el afirmar que el movimiento rectilíneo es el estado natural y por tanto no requiere una acción externa para mantenerlo, con el mismo derecho se podría proponer como natural otro tipo de movimiento (por ejemplo, el circular) y establecer que no se requiere una fuerza para mantenerlo sino sólo para modificarlo. En realidad, la argumentación de Laplace estaba basada en el principio epistemológico de que los estados no requieren explicación y los procesos sí que la requieren.

Una característica importante de la ley de la inercia reside en la dificultad (en realidad imposibilidad) de ser comprobada experimentalmente. Si la primera ley se refiere al movimiento de un cuerpo libre de acciones externas cualquier diseño experimental requeriría de un mecanismo para localizar en cada instante la posición del cuerpo y para medir el tiempo, pero, si todos los cuerpos se atraen entre si, según la ley de gravitación universal que acompaña a las leyes de la dinámica, el cuerpo en movimiento dejaría de estar libre 
de fuerzas externas y no se cumpliría la condición básica para poder llevar a cabo el experimento.

Se argumenta frecuentemente que la primera ley es también válida para cuerpos sometidos a varias fuerzas externas siempre que la resultante de estas fuerzas sea nula, sin embargo, el argumento resulta tautológico si no se proporciona un método alternativo para comprobar que la resultante de estas fuerzas es nula que no sea por el mantenimiento del estado de reposo o movimiento rectilíneo y uniforme del cuerpo. Por lo tanto, como señaló Reech (1852), ya que no estamos en condiciones de determinar experimentalmente la trayectoria y la velocidad que seguiría un cuerpo libre de interacciones, la dinámica newtoniana elige, convencionalmente, que el cuerpo seguiría un movimiento rectilíneo y uniforme (incluyendo el reposo como caso particular).

Según Hanson (1958), aunque la ley de la inercia fue inicialmente propuesta como una extrapolación experimental del comportamiento de cuerpos que deslizan sobre planos poco rugosos (per inductionem collectae), adquirió con los años la consideración de convención (o de enunciado a priori). La primera ley nos habla de lo que ocurrriría en la naturaleza si se dieran ciertas condiciones. En este mismo sentido, se ha criticado la distinción que hizo Newton entre hipótesis y axiomas, ya que la ley de la inercia debería aparecer entre las hipótesis y no entre los axiomas (o leyes). Un "cuerpo libre" se mueve (por definición) con movimiento rectilíneo y uniforme (incluye el reposo), y si deja de cumplirse alguna de estas condiciones (no rectilí- neo o no uniforme) es indicio de que está actuando sobre él alguna fuerza.

Otro de los aspectos problemáticos que dificulta la interpretación actual de la primera ley radica en que Newton partía de la hipótesis de espacio y tiempo absoluto: un sistema de referencia fijo para hacer las mediciones. Pero si no aceptamos como válida la hipótesis de espacio y tiempo absoluto, la afirmación que establece que un cuerpo libre recorre espacios iguales en tiempos iguales carece de sentido. En decir, sin el establecimiento de un sistema de referencia apropiado la primera ley no aporta ninguna información.

Una interpretación actual de la primera ley es que la misma establece el marco en el que es válida la dinámica newtoniana para fuerzas de interacción (no ficticias): los sistemas de referencia inerciales. Se puede definir un sistema de referencia inercial como aquel en el que un "cuerpo libre" se movería con movimiento rectilíneo y uniforme. Este marco inercial permitiría definir la escala de tiempos en base a los intervalos iguales recorridos por partículas libres.

Bridgman (1961), a mediados del siglo pasado, elaboró las siguente construcción mental de un marco inercial. "Un sistema de tres ejes ortogonales rígidos determina un sistema de referencia inercial si tres partículas no sometidas a ninguna fuerza que se lanzan a lo largo de los tres ejes con velocidades arbitrarias, continúan moviéndose a lo largo de los mismos con las mismas velocidades iniciales". Aunque nuestros laboratorios terrestres no pueden ser considerados marcos de referencia de este tipo, sí que es posible medir cuánto 
se desvían del comportamiento requerido las tres masas que se lanzan en cada eje e incorporar estas desviaciones en forma de correcciones. Desde este punto de vista, la primera ley definiría a los marcos inerciales en contraposición con los marcos no inerciales, proponiendo a los experimentalistas el problema de distinguir las fuerzas reales debidas a la interacción de los objetos, de las fuerzas ficticias o pseudofuerzas debidas a la aceleración del marco de referencia.

Una formulación actual de la primera ley podría ser: "En un sistema inercial toda partícula libre se mueve a velocidad constante". Entendiéndose como partícula libre aquella que no está sometida a ninguna fuerza. Añadiendo que las leyes básicas de la física son idénticas (invariantes) en todos los sistemas de referencia que se mueven con movimiento uniforme (velocidad constante) unos respecto a otros. Sin embargo, la primera ley se encuentra inevitablemente inmersa en el sistema de las otras leyes para poder entender el significado de "cuerpo libre".

\section{La segunda ley de Newton}

La segunda ley de los Principia de Newton establece que "el cambio de movimiento es proporcional a la fuerza motriz impresa, y se realiza en la dirección en la que actúa la fuerza impresa" ("Mutationem motus proportionalem esse vi motrici impressae, et fieri secundum lineam rectam qua vis illa imprimitur", Principia, p. 13). Entender la fuerza como la causa del cambio de movimiento y la proporcionalidad entre la fuerza impresa y el cambio de movimiento de un cuerpo es la esencia de esta segunda ley.

Newton entendía el término "movimiento" cercano a lo que actualmente entendemos por "cantidad de movimiento", es decir, una magnitud combinada de la masa y la velocidad $(m \bar{v})$. La expresión de "cambio de movimiento proporcional a la fuerza impresa $(\overline{\mathrm{F}})$ "se podría expresar en lenguaje matemático como: $\overline{\mathrm{F}} \Delta \mathrm{t}=\mathrm{m} \Delta \bar{v}$, que permitiría interpretar mejor, según Cohen (1979), el resultado de una fuerza continua (en lugar de cuasi-instantánea) y es más coherente con la concepción "fluxional" que Newton tenía de las fuerzas.

Un punto esencial en este axioma es la incorporación del concepto de masa (quantitae materiae) que se obtendría mediante la combinación de una magnitud intensiva: la densidad, y una extensiva: el volumen (Mashood, 2009). Aunque esta definición pudiera parecer circular, ya que la densidad no es más que la razón entre la masa y el volumen, la densidad puede determinarse alternativamente por medios hidrostáticos (aunque tácitamente se utilice la proporcionalidad entre masa y peso). Una vez establecida una masa de referencia se pueden cuantificar otras masas a partir de la comparación en una balanza de brazos iguales. Ahora bien, si la masa viene determinada por comparación con una masa patrón mediante la balanza de brazos (masa gravitacional) se restringe la definición operacional a cuerpos pequeños y no es extrapolable a masas como la de la Luna o la de la Tierra.

La noción newtoniana de fuerza viene caracterizada en la definición IV como: "fuerza es una acción ejercida 
sobre un cuerpo con el objeto de cambiar su estado de reposo o de movimiento uniforme en línea recta". Como bien ha señalado Coelho (2009),el hecho de que la fuerza impresa sea proporcional al cambio de movimiento ha sido aceptado por todos los físicos, sin embargo, en lo que no parece haber consenso es en utilizar para ello el término "causa". Lazare Carnot (Principes fondamentaux de l'équilibre et du mouvement, 1803, p.47) consideró que afirmar que la fuerza es "la causa del cambio de movimiento" es invocar una "noción metafísica y oscura que enturbia la claridad de la ciencia", y Ernest Mach (The science of mechanics, 1883), entre otros muchos, abogaba por que en el futuro la ciencia pudiera prescindir de las "perniciosas ideas de causa y efecto" y sustituirlas por las de una simple relación funcional.

Euler, en su Discovery of a New Principle of Mechanics (1750), propuso por primera vez la expresion de la fuerza como producto de la masa por la aceleración. La aceleración no presentaría mayores dificultades por ser un concepto cinemático y la masa, según Euler, sería el coeficiente de proporcionalidad entre la fuerza aplicada y la aceleración, por lo que se trasladaba el problema del concepto de masa al concepto de fuerza.

Mach (Ueber die Definition der Masse, 1868) sugirió que la segunda ley fuera presentada según la secuencia: aceleración - masa - fuerza, y propuso determinar la masa (inercial) a partir de la relación inversa de las aceleraciones de dos cuerpos que interactúan: $\mathrm{m}_{\mathrm{A}} /$ $\mathrm{m}_{\mathrm{B}}=-\mathrm{a}_{\mathrm{B}} / \mathrm{a}_{\mathrm{A}}$ ("the negative inverse ratio of the mutually induced accelerations of any two bodies called their mass ratio").
Sin embargo, esta definición, o más bien esta prescripción de cómo medir la masa, no está exenta de dificultades ya que: 1) incorpora implícitamente a la tercera ley de Newton para determinar la masa; 2) necesita la especificación del sistema de referencia en el que deben ser medidas las aceleraciones; 3 ) requeriría demostrar que la masa inercial (medida por este procedimiento) equivale a la masa gravitacional (medida a partir del peso o de la densidad).

La noción de fuerza es frecuentemente interpretada a partir de la sensación del esfuerzo muscular al empujar o tirar de un cuerpo, para pasar a ser cuantificada posteriormente mediante la deformación de un resorte. Reech (1852), elaboró su mecánica a partir de las mediciones de fuerzas en hilos elásticos o resortes que, al tiempo de indicar la existencia real de las fuerzas, permitían medir su intensidad por deformación (dinamómetro). Sin embargo, esta idea de fuerza de su "dinámica de hilos" ("dynamic of theards") ha sido fuertemente objetada, ya que perfila una idea de fuerza proveniente esencialmente de la estática, que es ajena a la dinámica de los cuerpos en movimiento, y está limitada a fuerzas de contacto y por tanto difícilmente extensible a los fenómenos que más interesan a la física: gravitatorios, eléctricos o magnéticos.

Kirchhoff pretendió en su Mechanics (1876) una reformulación tomando como punto de partida que la función esencial de las leyes no era la explicación sino la descripción del movimiento. Kirchhoff defendía que la fuerza era un mero constructo teórico formalmente igual al producto de la masa por la 
aceleración. Desde esta perspectiva, la segunda ley sería superflua y sería reemplazada por una simple definición. Una de sus principales objeciones a la teoría original de Newton consistía en que, aunque la aceleración de un objeto proporciona información de la fuerza resultante sobre el mismo, esta información no permite distinguir cuáles son las fuerzas actuantes.

El principio de superposición establece que si varias fuerzas actúan simultaneamente sobre un cuerpo, la fuerza resultante es igual a la suma vectorial de las fuerzas que actúan independientemente sobre el cuerpo (regla del paralelogramo). Este principio aparece incluido en los Principia de Newton como Corolario 1, después de la tercera ley, pero es requisito indispensable para la comprensión y aplicación de las leyes, así como para la caracterización vectorial de las fuerzas. Si bien es cierto que a partir de fuerzas actuantes se puede obtener la fuerza resultante, el recíproco no es cierto. Daniel Bernoulli (Examen principiorum mechanicae et demonstrationen geometricae de compositione et resolutione virium, 1726) intentó presentarlo como una verdad $a$ priori independiente de observaciones experimentales, pero Euler (Discovery of a New Principle of Mechanics, 1750) demostró claramente que no es posible demostrarlo analíticamente sin hacer otras suposiciones.

Si se opta por la definición cuantitativa de fuerza propuesta por Kirchhoff, como producto de la masa por la aceleración $\bar{F}=m \bar{a}$ el lado izquierdo de la ecuación podría no representar a ninguna fuerza en particular sino a la acción conjunta de todas las que están actuando sobre el cuerpo. Por lo tanto, la ecuación $\bar{F}=m \bar{a}$ representaría, en todo caso, sólo una de las características de la fuerza (resultante). Pero, si el movimiento está condicionado por varias fuerzas estas fuerzas no pueden ser caracterizadas ni determinadas separadamente a partir de esta ecuación.

Según Kirchhoff (1876) definiendo a la fuerza como la masa de un cuerpo por la aceleración, se podría hablar de "fuerzas de aceleración": X, Y, Z, en un sistema cartesiano, a las segundas derivadas de las coordenadas del cuerpo con respecto al tiempo (dejando momentáneamente de lado el coeficiente constante de la masa del cuerpo).

$$
\frac{\partial^{2} x}{\partial t^{2}}=X \quad \frac{\partial^{2} y}{\partial t^{2}}=Y \quad \frac{\partial^{2} z}{\partial t^{2}}=X
$$

La fuerza para Kirchhoff no tendría mayor significado que el nombre para la segunda derivada respecto al tiempo de las coordenadas de la posición $P(x, y, z)$ del cuerpo en un determinado marco de referencia. La pregunta evidente sería: ¿Porqué la segunda derivada y no la tercera, o la cuarta? Poincaré (1902) intentó responder al dilema anterior sosteniendo que son precisamente las derivadas segundas de las coordenadas las que su vez dependen de las posiciones del cuerpo respecto a las posiciones y características de los cuerpos cercanos, por el contrario, las derivadas de orden superior de las mismas no contribuyen a la simplificación de la descripción del movimiento. 
Así pues, la derivada segunda de las coordenadas de un cuerpo (la aceleración) es la que está determinada por la posición del objeto que se mueve respecto al resto de los cuerpos del universo. La ecuación de movimiento de una masa $\mathrm{m}$ sobre la que actúa una fuerza $\overline{\mathrm{F}}$, tiene soluciones no triviales $(\bar{r} \neq$ constante) incluso en los casos en los que el término de fuerza sea nulo.

$$
\bar{F}=m \frac{d^{2} \bar{r}}{d t^{2}}
$$

Como ya objetó Nielsen (1929), si se define fuerza por la ecuación $\bar{F}=m \bar{a}$, ambos miembros de la ecuación deberían tener el mismo significado, sin embargo, el término de la izquierda: la fuerza, representa la acción del resto del universo sobre la masa $m$, mientra el término de la derecha: la masa por su aceleración, representa el cambio de movimiento en respuesta a la fuerza actuante. No es un simple cambio de variable ni una definición, el significado físico del primer miembro es radicalmente diverso del significado del segundo miembro, y es este hecho el que otorga importancia al producto de la masa por aceleración y justifica la introducción de un nombre especial para él.

\section{La tercera ley de Newton}

Newton en su tercera ley establece que: "Para cada acción existe siempre una reacción, y las acciones mutuas entre dos cuerpos son siempre iguales y en dirección contraria" "Actioni contrariam semper et æqualem esse reac- tionem: sive corporum duorum actiones in se mutuo semper esse æquales et in partes contrarias dirigi”, Principia, p.13). La tercera ley introduce una de las características fundamentales del concepto de fuerza y es el de interacción entre los cuerpos. Las fuerzas siempre están referidas a dos cuerpos A y B, cada uno de los cuales ejerce una fuerza sobre el otro de igual magnitud y sentido contrario.

$$
\bar{F}_{A \rightarrow B}=-\overline{\mathrm{F}}_{\mathrm{B} \rightarrow \mathrm{A}}
$$

Esta ley no ha sufrido grandes modificaciones en la interpretación actual, incluso introduciendo los campos de fuerzas, hasta llegar a los conceptos de relatividad especial. Existen unas limitaciones inherentes a la validez de esta tercera ley: hoy sabemos que todas las señales o fuerzas tienen una velocidad de propagación finita. Entre las "hipótesis" implícitas de la formulación de los Principia las fuerzas se ejercían a distancia e instantáneamente. La tercera ley establece que $\overline{\mathrm{F}}_{\mathrm{A} \rightarrow \mathrm{B}}$ es igual y opuesta a $\overline{\mathrm{F}}_{\mathrm{B} \rightarrow \mathrm{A}}$ cuando ambas se miden en le mismo instante. Este requisito no está de acuerdo con el intervalo finito de tiempo que se necesita para que una partícula "perciba" la fuerza que la segunda partícula ejerce sobre ella. En los choques entre automóviles es una aproximación excelente pero en los choques atómicos la tercera ley no es una buena aproximación. Curiosamente, aunque en su obra Newton menciona frecuentemente la fuerza de inercia, no señala cuál pudiera ser la fuerza de reacción asociada. 
La tercera ley puede ser analizada a partir de la conservación de la cantidad de movimiento o momento lineal $\bar{p}=m \bar{v}$ de un sistema de dos particulas y de la definición de fuerza como derivada de la cantidad de movimiento respecto al tiempo. Sean A y B las partículas de respectivas cantidades de movimiento: $\overline{\mathrm{p}}_{\mathrm{A}} \mathrm{y} \quad \overline{\mathrm{p}}_{\mathrm{B}}$. Si es sistema es aislado $\overline{\mathrm{p}}_{\mathrm{A}}+$ $\overline{\mathrm{p}}_{\mathrm{B}}=$ constante vectorial, $\mathrm{y}$ por lo tanto:

$$
\frac{d \bar{p}_{A}}{d t}+\frac{d \bar{p}_{B}}{d t}=0,
$$

y entonces

$$
\frac{d \bar{p}_{B}}{d t}=-\frac{d \bar{p}_{A}}{d t} \quad \text { y } \quad \bar{F}_{A \rightarrow B}=-\overline{\mathrm{F}}_{\mathrm{B} \rightarrow \mathrm{A}}
$$

Desde esta perspectiva, la tercera ley es una consecuencia de la conservación del momento lineal en un sistema aislado de dos partículas, cuya validez se extiende hasta la mecánica cuántica. Sin embargo, la validez de la conservación del momento es puesta en entredicho cuando se observan interacciones electromagnéticas entre partículas, aunque la conservación es salvaguardada sólo si se considera que la interacción entre las partículas tiene como intermediario el campo electromagnético, el momento lineal aparentemente faltante corresponde al momento de radiación del campo electromagnético.

\section{La ley de gravitación universal}

Newton introduce la ley de gravitación en el Libro III de sus Principia, la cual resulta un complemento fundamental de las leyes generales de la dinámica establecidas en los Libros I $y$ II. A partir del análisis de los resultados empíricos del movimiento de los planetas obtenidos por Kepler, Newton concluye que "las fuerzas centrípetas varían inversamente con el cuadrado de los radios" y, a partir de los valores de la las masas de la Tierra y la Luna, concluye que "la fuerza atractiva sería directamente proporcional a las masas e inversamente proporcional al cuadrado de las distancias".

Newton desarrolló las consecuencias que dicho tipo de fuerza tenía para la explicación del movimiento de planetas y cometas, para el efecto de las mareas y para la precesión de los equinoccios. La propuesta newtoniana reconoce no haber logrado encontrar las causas de la existencia de esta fuerza gravitatoria, pero descarta las explicaciones mecánicas de vórtices o del impacto de partículas del éter circundante para explicar el origen de la fuerza gravitatoria. La gran importancia de su propuesta es aportar un modelo de fuerza de interacción "a distancia"que permite obtener resultados de la ecuación diferencial coherentes con las observaciones:

$$
\bar{F}=m \frac{d^{2} \bar{r}}{d t^{2}} \quad \rightarrow \quad-G \frac{M m}{\bar{r}^{2}} \hat{r}=m \frac{d^{2} \bar{r}}{d t^{2}}
$$

Los años posteriores permitieron ampliar el espectro de fuerzas en la ecuación diferencial anterior incorporando las fuerzas electricas, magnéticas.

\section{Algunos comentarios a la revisión histórica de las Leyes de Newton}

Como hemos visto, las leyes de Newton en su desarrollo histórico han sido 
sometidas a múltiples observaciones importantes. Algunas de las observaciones provienen de planteamientos positivistas pretendiendo definiciones explícitas de los términos de la teoría y una metodología de comprobación experimental de cada una de las leyes (Mach, Reech, etc.), mientras otras críticas son de matiz convencionalista que no han vacilado en declarar a las leyes de la mecánica como "a priori" o analíticas (Poincaré, Duhem, etc).

La posición preponderante hoy día es que cada una de las leyes no debe ser analizada como un enunciado aislado, sino como parte de la propuesta general, similar a los postulados de Euclides en la geometría que, al parecer, fue la propuesta que Newton tomó como modelo para elaborar su teoría del análisis de los fenómenos físicos. Desde esta perspectiva, los conceptos de fuerza y de masa estarían definidos implicitamente por las leyes, de la misma manera que las nociones de punto, línea recta o plano, estan definidos en los axiomas euclideanos al establecer las relaciones con los otros términos de la teoría. Los axiomas (leyes) de Newton constituirían en los Principia un apartado diferente de las definiciones, los corolarios o las fórmulas, porque no necesitarían ser probados, aunque esto no quiere decir que no tengan una clara interpretación experimental.

Una "transposición didáctica" adecuada debería tener en cuenta: 1) que las leyes de la dinámica fueron enunciadas hace varios siglos, y presentarlas en su versión original significa ignorar las modificaciones profundas que el pensamiento físico a experimentado en los últimos siglos; 2) que la presentación de las leyes debe evitar perspectivas epistemológicas que configuren una visión distorsionada de la ciencia y de la actividad científica; 3) que aunque los estudiantes no llegan a la situación de enseñanza con la mente "tamquam tabula rasa in qua nihil est scriptum" (como una tabla lisa en la que nada está escrito), serán los nuevos sistemas de representación proporcionados por los profesores y los libros de texto los que les permitirán reelaborar sus interpretaciones, aunque las anteriores sigan siendo utilizadas en contextos particulares (Pozo y Gomez Crespo, 1998).

\section{La presentación de las leyes de newton en los textos de enseñanza}

La mayoría de los libros de texto expone las leyes de Newton partiendo de una breve introducción histórica e introduciendo "intuitivamente" los conceptos de masa, y de fuerza. Algunos textos utilizan el planteamiento causal de la dinámica: "la segunda ley de Newton relaciona la descripción del movimiento con la causa del mismo, la fuerza" (Giancoli, 1988, p.68). Sin embargo, la mayoría evita la utilización de un término problemático como"causa" en la caracterización de la dinámica: "el propósito de la mecánica clásica es establecer una conexión entre el movimiento de un cuerpo y las fuerzas que actúan sobre él" (Serway, 1993, p.98), o "la dinámica es la parte de la mecánica que estudia conjuntamente el movimiento y las fuerzas que lo originan" (Sears, Zemansky y Young, 1986, p.70). 
Los libros de Tipler, 1976; Roller y Blum, 1983; Sears, Zemansky y Young 1986, entre otros, transcriben las leyes en la versión original de Newton (incluso en latín), seguidas de una versión actualizada de las mismas. La actualización consiste básicamente en sustituir el término "fuerza impresa" por: a) "fuerza externa" ; b) "fuerza neta"; o, c) "fuerza de desequilibrio externa".

El libro de Sears, Zemasnky y Young, (1986, p.70) presenta la primera ley como un caso particular de la segunda: "cuando la fuerza resultante que actúa sobre un cuerpo es cero la aceleración también lo es". Para expresar la condición de cuerpo ausente de fuerzas actuantes, algunos libros prefieren introducir, antes del enunciado de la primera ley, el concepto de "cuerpo libre" y enunciar la primera ley de la forma: "una partícula libre se mueve siempre con velocidad constante" (Alonso y Finn, 1986, p.156) o "cuando un cuerpo se deja aislado, mantiene una velocidad constante" (Fishbane, Gasiorowicz y Thorton, 1994; Roller y Blum, 1983).

La primera ley es presentada en la mayoría de los textos como un extrapolación experimental. Partiendo del experimento de Galileo con planos inclinados (Giancoli, 1988, p.67), o simplemente proponiendo un diseño experimental en un plano horizontal pulido: "si pudiéramos eliminar por completo el rozamiento no se necesitaría ninguna fuerza para mantener en movimiento el cuerpo" (Sears, Zemansky y Young, 1986, p.26).

En todos los textos se asoma la dificultad inherente a la necesidad de un marco de referencia para poder afirmar si el cuerpo está en reposo o movimiento: "sólo se puede decir que un cuerpo está en reposo según un marco determinado y no en sentido absoluto" (Fishbane, Gasiorowicz y Thorton, 1994, p.95). "El sistema de referencia inercial es aquel en el que es válida la primera ley de Newton" (Serway, 1993, p.102). Se resalta con frecuencia que existen infinitos sistemas de referencia inerciales y se menciona al sistema de referencia de las estrellas fijas como marco de referencia inercial y que la Tierra, a pesar de su rotación, puede ser considerada con cierta aproximación un marco de referencia inercial. Ocasionalmente (Kittel, Knight y Ruderman, 1968) se apunta la propuesta relativista de que no existe ningún sistema de referencia absoluto en el universo, pero "el sistema de referencia en el que la primera ley de Newton se cumple de modo más exacto consiste en un sistema de coordenadas rígido que se mueve con una velocidad constante relativa a las estrellas fijas. Este sistema de referencia se llama sistema inercial primario" (Roller y Blum, 1983, p. 101).

La segunda ley en los libros adopta frecuentemente la forma de: "la aceleración de un objeto es directamente proporcional a la fuerza resultante que actúa sobre él e inversamente proporcional a la masa" (Serway,1993, p.103) con la puntualización de que "la dirección de la aceleración se encuentra en la dirección de la fuera neta aplicada" (Giancoli, 1988, p.67).

La presentación de la segunda ley incluye los conceptos de fuerza y masa. Para caracterizar al concepto de fuerza se parte, en ocasiones, de la definición tomada de los Principia de Newton como "la acción ejercida sobre un cuer- 
po con el objeto de cambiar su estado de reposo o de movimiento uniforme en línea recta" (Roller, Blum, 1983, p.102). Pero, como esta definición es básicamente una reescritura de la Primera ley, se prefiere en los textos interpretar a la fuerza como una medida de la interacción entre dos cuerpos o entre un cuerpo y su entorno. Se parte, normalmente, de una interpretación intuitiva como la sensación muscular al empujar o tirar de un cuerpo, para pasar a la forma cuantificable del resorte (dinamómetro). No se aclara normalmente la relación entre este tipo de fuerzas de contacto, definidas por deformación de un cuerpo elástico, y las fuerzas como la de la gravitación, eléctrica y magnética que actúan a distancia.

La masa es definida habitualmente, a la manera newtoniana, como cantidad de materia o medida de la inercia (Eisberg y Lerner, 1981), y se determina mediante la comparación con una masa patrón en la balanza de brazos. La masa inercial es operativamente determinada (con un método similar al de Mach) aplicando una misma fuerza a objetos de diferente masa, por lo que $m_{1} a_{1}=m_{2} a_{2}$ y por lo tanto la razón de las masas sería inversa a la razón de las aceleraciones: $\frac{m_{1}}{m_{2}}=\frac{a_{2}}{a_{1}}$. Basta elegir una masa patrón para determinar el valor de cualquier masa inercial. Podría objetarse que se está utilizando implícitamente la segunda ley de Newton para definir la masa, por lo que persiste cierta circularidad en el argumento. Los libros que hacen una presentación de la dinámica asociada a la conservación de la cantidad de movimiento (Alonso y Finn, 1986, Eisberg y Lerner, 1981; Kittel, Knight y Ruderman, 1968; Roller y Blum, 1983) utilizan una estrategia que es inherente a la conservación del momento lineal: en una interacción entre dos cuerpos de masas $\mathrm{m}_{1} \mathrm{y} \mathrm{m}_{2}$, la conservación de la cantidad de movimiento conlleva a que $m_{1} \Delta v_{1}+m_{2} \Delta v_{2}$ $=0 \mathrm{y}$ el cociente de las masas será: $\frac{m_{1}}{m_{2}}=-\frac{\Delta v_{2}}{\Delta v_{1}}$, donde el segundo miembro es el cociente de las variaciones de velocidad de cada cuerpo después de la interacción. Hay que hacer notar que en este caso se estaría utilizando la tercera ley de Newton para obtener una definición operativa de masa inercial.

Una vez "definidos" los conceptos de fuerza y de masa, la segunda ley se puede escribir como que la aceleración es directamente proporcional a la fuerza aplicada e inversamente proporcional a la masa del cuerpo $\bar{a}=\frac{\bar{F}}{m}$ (Blatt, 1991, Lea y Burke, 1999). Aunque también viene escrita en su forma más práctica como: $\bar{F}=m a \bar{~}$. Esta última expresión se toma en ocasiones en el sentido de Euler-Kirchhoff como definición de fuerza: " $\bar{F}=m \bar{a}$ define la fuerza" (Meriam, 1992, p.5), pero habitualmente se prefiere presentar como una extrapolación experimental utilizando cuerpos de diferentes masas sometidos a una fuerza calibrada con un dinamómetro.

Los textos de Eisberg y Lerner (1981), Alonso y Finn (1986), Kittel, Knight y Ruderman (1968), Roller y Blum (1983), entre otros, prefieren partir del principio de conservación de 
cantidad de movimiento: postulando que "la cantidad de movimiento de una partícula o sistema aislado se conserva, es decir, no varía ni en módulo ni en dirección con el paso del tiempo". La fuerza es definida como la derivada de la cantidad de movimiento respecto al tiempo, incluyendo fuerzas en sistemas de masa variable y afirmando que "la fuerza es igual al producto de la masa por la aceleración sólo si la masa es constante" (Alonso, Finn, 1986, p.165).

La tercera ley de Newton se presenta en los libros de texto en muchos casos como principio de acción y reacción: "a toda acción se opone una reacción igual y dirigida en sentido contrario" (Resnick, Halliday, 1980, p. 92). Se aclara que "las fuerzas se presentan siempre por pares" o, consecuentementemente, que "no puede existir una fuerza aislada" (Serway, 1993, p. 106). La tercera ley implica siempre dos cuerpos distintos y separados (Blatt, 1991, p. 60), pero los múltiples ejemplos incluidos en los textos no terminan de aclarar este punto, quizás porqué enfatizan el llamado "diagrama del cuerpo libre", que al centrarse en una masa sola no figuran las fuerzas de reacción. Para los que parten del principio de conservación del momentum "la tercera ley es (simplemente) una consecuencia de la definición del principio de conservación y de la definición de fuerza" (Alonso y Finn, 1986, p.164).

\section{Algunas consecuencias para la enseñanza}

El propósito del presente este trabajo ha sido revisar algunos aspectos en la enseñanza de la dinámica clásica con el objeto de contribuir al análisis del contenido curricular existente que permita analizar su enfoque, coherencia y actualización (entendida como la posibilidad de incorporar los conceptos de la mecánica del Siglo XX), así como ayudar a los profesores a ser conscientes de las dificultades inherentes al tema.

En base al análisis realizado de una muestra de libros de texto, tomando como marco teórico la revisión histórica de las Leyes de Newton, resaltan tres principales perspectivas de presentación de la dinámica clásica, aunque cada una de ellas con diversas variantes y matices: la perspectiva A: basada en la propuesta original de Newton, con las aportaciones de Mach, y que llamaremos "cuadro de Newton-Mach"; la perspectiva B: también basada esencialmente en la propuesta de Newton, pero aceptando la propuesta de Kirchhoff de tomar como definición de fuerza el producto de la masa por aceleración: "cuadro de Newton-Kirchhoff”, y la propuesta C: basada el principio de conservación del momento lineal, y definida la fuerza como derivada del momento lineal, incluyendo en la segunda ley la dinámica de sistemas de masa variable , y que, a falta de una denominación mejor, llamaremos "cuadro de Momento lineal". De estas tres perspectivas, esquemas o cuadros, los libros de texto analizados serían agrupables por sus características mas destacadas en alguna de ellas, aunque esto signifique en ocasiones una simplificación excesiva.

A) Cuadro de Newton-Mach. Esta perspectiva parte de los enunciados originales de Newton e incorpora las aportaciones de Mach para definir operacionalmente la masa, se define opera- 
cionalmente la fuerza mediante la deformación de cuerpos elásticos y presenta la segunda ley de Newton como resultado experimental de proporcionalidad entre la fuerza y la aceleración, siendo la masa inercial la constante de proporcionalidad. Normalmente introduce el concepto de masa gravitacional como cantidad de materia, a la manera de Newton, y lo determina con la balanza de brazos iguales (lo que puede dificultar su extensión a los conceptos de masa en mecánica cuántica y relatividad, donde deja se ser un parámetro constante). La definición de masa inercial está basada la propuesta operacional de Mach de que la razón entre dos masas que interactúan es inversamente proporcional a la razón aceleraciones que adquieren (este elemento es quizás el elemento más extendido en los libros de texto, sin aclarar que se está utilizando implícitamente la tercera ley para definir la masa). En este marco se utiliza el referente histórico para el desarrollo de las leyes de la dinámica y hace referencia al trabajo de Galileo para la primera ley, y al de Kepler acerca de la ley de gravitación. Este cuadro comparte un enfoque esencialmente positivista (en ocasiones operacionalista) del esquema conceptual de la dinámica, valorando el origen empírico de los enunciados legales y el que de la teoría provenga de la generalización de los fenómenos observados. Aspira a una definición independiente de los términos utilizados: fuerza, masa, etc., y utiliza raramente conceptos de la física actual como: sistema de partículas, campo gravitacional, espacio o tiempo relativo, etc. Aunque varios de los textos analizados responden a este cuadro con mayor o menor exactitud podríamos citar entre ellos a los textos de Resnick y Halliday (1980), Sears, Zemansky y Young (1986).

B) Cuadro de Newton-Kirkhhoff. Esta perspectiva adopta un sistema mixto de presentación de la dinámica. Parte de los enunciados de Newton, pero enfatiza que la verdadera definición de fuerza es el producto de la masa por la aceleración: $\bar{F}=m \bar{a}$. El formalismo de la mecánica clásica se convierte en axiomático en el sentido que todos los resultados se siguen de la definición de fuerza, más bien que de leyes deducidas experimentalmente. La primera ley, desde esta perspectiva, es un caso particular de la segunda, ya que "no añade nada nuevo a la descripción del movimiento, pero se incluye porque formaba parte de los enunciados clásicos de Newton" (Meriam, 1992, p.5). Es importante, sin embargo, que exista siempre correspondencia entre las observaciones del mundo real y los resultados obtenidos con el formalismo de la dinámica. En el caso en que las observaciones se hacen desde sistemas no inerciales las mediciones no coinciden con los resultados derivados del formalismo, este criterio sirve para distinguir los sistemas inerciales de los no inerciales, y para introducir fuerzas ficticias. Este planteamiento está más próximo de las corrientes convencionalistas. Es más frecuente en libros especializados que en los de física introductoria. La importancia de la historia desde esta perspectiva es menor y sobre todo anecdótica. Es el planteamiento que siguen esencialmente, aparte de Meriam (1992), algunos libros de mecánica clásica (Goldstein, 1972). 
C) Cuadro de Momento lineal. Esta perspectiva está basada en la conservación del momento lineal o cantidad de movimiento. Postula el principio de conservación del momento lineal en sistemas aislados. Se adopta la definición convencional de la fuerza como derivada del momentum lineal. La primera ley, desde esta perspectiva, es la expresión de la conservación del momento lineal para la partícula aislada, la segunda ley es la definición de fuerza (que incluye casos de masa variable, incluso cuando la masa cambia con la velocidad, como en los casos relativistas). La tercera ley es presentada como un caso particular del principio de conservación de momento lineal para un sistema aislado de dos partículas. Este tipo de interpretación es básicamente convencionalista (como corresponde a todos los enfoques a partir de leyes de conservación) según el cual los axiomas son valorados por los resultados que producen sin requerir una extrapolación a partir de experimentos en el mundo real. No incorpora habitualmente elementos históricos en su presentación. La noción de sistema de partículas es inherente a su estructura, y el esquema de ley de conservación resulta válido en muchos aspectos de la física moderna y la teoría clásica de campos, en los que el concepto de fuerza queda difuminado. La formulación de la tercera ley de Newton mediante este cuadro permite posteriormente su aplicación a los casos de interacciones electromagnéticas. Entre los libros de texto que siguen esta perspectiva se encuentran, entre otros: Alonso y Finn, 1986; Kittel, Knight y Ruderman, 1968; Roller y Blum, 1983.
El problema de la presentación del contenido de la mecánica clásica es complejo. Hemos pretendido en este trabajo mostrar que de hecho se vienen siguiendo diferentes estrategias, con resultados posiblemente diversos. La coherencia de la presentación parece ser mayor en los esquemas convencionalistas, ya que las leyes de Newton no son demostrables (stricto sensu) experimentalmente. Tratándose de la presentación de la mecánica clásica, no se pretende una actualización a las mecánicas del siglo XX (cuántica o relativista), sino que permita un extensión posterior, no contradictoria con sus postulados básicos. Desde este punto de vista, la perspectiva de conservación de la cantidad de movimiento resulta más abierta a las aportaciones posteriores. No está dicho que una de las anteriores perspectivas que hemos caracterizado sea la más adecuada, ya que dependerá de otros factores, sobre todo del nivel y tipo de estudiantes a los que va dirigido el material. Sin embargo, una reflexión amplia y profunda sobre estos supuestos nos permitiría diseñar una mejor "transposición didáctica" de la dinámica clásica o seleccionar la que pueda resultar más adecuada a nuestros propósitos, redundando en una mejora de comprensión de un tema fundamental tanto para la formación de científicos e ingenieros como para la formación cultural del ciudadano común.

\section{Referencias bibliográficas}

ALONSO, M. y FINN, E. (1986), Fisica Vol 1: Mecanica, Fondo Educativo Interamericano, Mexico. 
BERNOULLI, D. (1726), Examen principiorum mechanicae, et demonstrations geometricae de compositione et resolutione virium. Commentarii Academiae Scientarum Imperialis Petropolitanae, vol. 1, pp. 126-42.

BLATT, F. J. (1991), Fundamentos de Física. Prentice Hall Hispanoamericana, S. A. México.

BRIDGMAN, P.W. (1961), Significance of the Mach Principle, American JournalofPhysics, vol.29(1),pp.32-36. http://dx.doi.org/10.1119/1.1937666

CARRASCOSA, J. y GIL PÉREZ, D. (1992), Concepciones alternativas en Mecánica, Enseñanza de las Ciencias, vol.10 (3), pp. 314-328.

CARNOT, L. (1803), Principes fondamentaux de l'équilibre et du mouvement, Deterville, Paris.

CHAMPAGNE, A. B., GUNSTONE, R. F. y KLOPFER, L. E. (1985), Effecting changes in cognitive structures among physics students. En West L. and Pines A. (eds.) Cognitive Structure and Conceptual Change, Academic Press.

CHEVALLARD, Y. y JOSHUA, M.A. (1991), La Transposition didactique $d u$ savoir savant au savoir enseigné, La Pensée Sauvage éditions, Grenoble.

COELHO, R.L. (2009), On the Concept of Force: How understanding its history can improve physics teaching. Science \& Education, vol.19 (1), pp. 91-113. http://dx.doi.org/10.1007/ s11191-008-9183-1
COHEN, B., (1979), La historia y el filósofo de la ciencia. En Suppe F. (ed.) La estructura de las teorias científicas, pp. 349-404, Editora Nacional, Madrid.

DRIVER, R., GUESNE, E. y TIBERGHIEN, A. (1985), Children's ideas in science. Milton Keynes: Open University Press.

DUIT, R. (2003), Conceptual Change: A Powerful Framework For Improving Science Teaching And Learning. International Journal of Science Education, vol. 25 (6), pp. 671-688. http://dx.doi. org/10.1080/09500690305016

EISBERG, R. M. y LERNER, L. S. (1981), Physics: Foundations and Applications: Vol. 1. McGraw Hill Book Company. New York.

EULER, L. (1736), Mechanics or the Science of Motion Presented Analytically. En Opera Omnia, Serie II, vol. 1-2. Teubner. Leipzig (1912).

EULER, L. (1750), Discovery of a New Principle of Mechanics. En Opera Omnia, Serie II, vol 5. Teubner, Leipzig (1912).

FISHBANE, P.M., GASIOROWICZ, S. y THORNTON, S.T. (1994), Fisica para Ciencias e Ingeniería: Vol 1. Prentice Hall Hispanoamericana, S. A. México.

FURIÓ, C., SOLBES, J. y CARRASCOSA, J. (2006), Las ideas alternativas sobre conceptos científicos: tres décadas de investigación. Alambique, vol. 48, pp. 64-77. 
GIANCOLI, D.C. (1988), Física General: Vol 1. Prentice Hall Hispanoamericana. México.

GIL PÉREZ, D. (1996), New trends in science education. International Journal of Science Education. vol. 18 (8), pp. 889-901. http://dx.doi. org/10.1080/0950069960180802

GOLDSTEIN, H. (1972), Mecánica Clásica. Editorial Aguilar. Madrid.

HANSON, N.R. (1958), Patterns of discovery. Cambridge University Press. Cambridge.

HESTENES, D. (1999), New Foundations for Classical Mechanics, Kluwer Academic Publishers N.Y.

JACOBI K.G. (1847), En H. Pulte (ed.) Vorlesungen über analytische Mechanik, Berlin 1847/48, Vieweg, Braunschweig, Wiesbaden, (1996).

KIRCHHOFF, G. (1897), Vorlesungen über Mathematische Physik, vol. I, $4^{\text {th }}$ edn, Teubner, Leipzig.

KITTEL, C., KNIGHT, W.D. y RUDERMAN, M.A. (1968), Berkeley Physics Course: Vol 1.Mecánica, Editorial Reverté, Barcelona.

LAPLACE, P. S. (1799), Traité de Mécanique Céleste, vol. I, Paris: Culture et Civilisation.

LEA, S.M., y BURKE, J.R. (1999), La naturaleza de las cosas: Vol 1, International Thomson Editores, México.

MACH,E.(1868),Ueber die definition der masse. Repertorium ExperimentalPhysik, vol. 4, pp. 355-359.
MACH, E. (1883), The science of mechanics, The open court publishing Co., Londres (1919).

MASHOOD, K. K. (2009), HistoricoCritical Analysis of the Concept of Mass: From Antiquity to Newton,

Proceedings of Episteme 3: International Conference to Review Research in Science, Technology and Mathematics Education, Mumbai, India.

MERIAM, J.L. (1992), Dinámica, Editorial Reverté, Barcelona

NEWTON, I. (1687), Isaac Newton's Philosophiae naturalis Principia Mathematica, En Koyrè A.y Cohen I.B. (eds.), Harvard Univ. Press, $3^{\text {rd }}$ (1975).

NIELSEN, J.R. (1929), On the concept of mass and force, School Science and Mathematics. vol. 29(1), pp. 59-64. http://dx.doi.org/10.1111/j. 1949-8594.1929.tb00616.x

POINCARÉ, J.H. (1902). La science et l'hypothèse, Flammarion, Paris.

POSNER, G.J., STRIKE, K.A., HEWSON, P.W. y GERTZOG, W.A. (1982), Accommodation of a scientific conception: Toward a theory of conceptual change, Science Education, vol.66, pp. 211-227. http://dx.doi.org/10.1002/ sce.3730660207

POZO, J. I. y GOMEZ CRESPO, M.A. (1998), El aprendizaje de conceptos científicos: del aprendizaje significativo al cambio conceptual, Aprender y enseñar Ciencia, Morata-MEC, Madrid. 
REECH, F. (1852), Cours de Mécanique d'après la nature généralement flexible et élastique des corps. CarilianGoeury et $V^{\text {or }}$ Dalmont, Paris.

RESNICK, R. y HALLIDAY, D. (1980), Fisica: Parte 1, Editorial Continental, México.

ROLLER, D.E. y BLUM, R. (1983), Física: Volumen 1, Editorial Reverté, Barcelona.

SERWAY, R.A. (1993), Física: Tomo 1, Mc Graw Hill, México.

SEARS, F.W., ZEMANSKY, M.W. y YOUNG, H.D. (1986), Física Universitaria, Fondo Educativo Latinoamericano, México.
SOLBES, J. y PALOMAR, R. (2011). ¿Por qué resulta tan difícil la comprensión de la Astronomía a los estudiantes? Didáctica de las ciencias experimentales y sociales, 25, 187211. http://ojs.uv.es/index.php/dces/ article/view/2370/1926

THIJS, G.D. y VAN DER BERG, E. (1995), Cultural factors in the origin and the remediation of alternative conceptions in physics, Science \& Education, vol. 4 (4), pp.317347. http://dx.doi.org/10.1007/ BF00487756

TIPLER, P.A. (1976) Física: Vol 1, Editorial Reverté, Barcelona. 
